\title{
Effective use of small-interfering RNA to characterize residual B-cell non-Hodgkin lymphoma cells following chemotherapy
}

\author{
I an K. T. Kusao ${ }^{1}$, David L. Troelstrup ${ }^{2}$, Melissa A. Agsalda ${ }^{2}$, Bruce T. Shiramizu ${ }^{2,3}$ \\ 1. Department of Reproductive Biology, University of Hawaii J ohn A. Burns School of Medicine, Honolulu, Hawaii, USA. \\ 2. Hawaii Center for AIDS, University of Hawaii John A. Burns School of Medicine, Honolulu, Hawaii, USA. 3. Department \\ of Pediatrics, Hematology/Oncology, University of Hawaii John A. Burns School of Medicine, Honolulu, Hawaii, USA
}

Correspondence: Bruce Shiramizu. Address: University of Hawaii, 651 Ilalo Street, BSB 325AA, Honolulu, Hawaii, USA, 96813. Telephone: 1-808-692-1677. Fax: 1-808-692-1984. E-mail: bshirami@hawaii.edu

Received: November 30, 2011 Accepted: January 18, $2012 \quad$ Published: March 1, 2012

DOI : 10.5430/jhm.v2n1p5 URL: http://dx.doi.org/10.5430/jhm.v2n1p5

\section{Abstract}

Background: Children diagnosed with non-Hodgkin lymphoma (NHL) respond well to therapy resulting in relatively good prognosis. The exceptions are those who continue to have minimal residual disease (MRD). MRD NHL cells have been characterized as having increased mitochondrial DNA (mtDNA) copy numbers with increased expression of citrate synthase and isocitrate dehydrogenase. A proof-of-concept was designed to use small-interfering RNA (siRNA) as a tool to elucidate the relationship between citrate synthase and isocitrate dehydrogenase with cancer cell integrity.

Methods: mtDNA copy number and lactate dehydrogenase activities were assessed in chemotherapy-exposed residual NHL cells after introduction of siRNA against citrate synthase and/or isocitrate dehydrogenase expression.

Results: There was a significant decrease in lactate production in cells transfected with citrate synthase siRNA ( $P=0.02)$. Citrate synthase-silenced cells had decreased mtDNA copy numbers $(P=0.03)$ compared to isocitrate dehydrogenasesilenced cells or combined citrate synthase- and isocitrate dehydrogenase-silenced cells.

Conclusion: Inhibition of citrate synthase expression in siRNA-treated NHL cells resulted in decreased mtDNA copy numbers and lactate dehydrogenase expression. This observation needs further validation to determine the role of citrate synthase in mtDNA integrity and if citrate synthase siRNA could be a potential therapeutic modality in eradicating residual B-NHL cells.

\section{Key words}

Non-Hodgkin lymphoma, Small-interfering RNA, Minimal residual disease

\section{I ntroduction}

Treatment for hematologic and lymphoid malignancies such as acute lymphoblastic leukemia (ALL) and non-Hodgkin lymphoma (NHL) has resulted in improved prognosis for children and adolescents ${ }^{[1]}$. However, following completion of therapy, continued presence of malignant cells, minimal residual disease (MRD), places patients with ALL and NHL at risk for relapse ${ }^{[2,3]}$. While advances have been made in earlier and more efficient detection of MRD, challenges continue Published by Sciedu Press 
to exist in eradicating residual tumor cells completely and improving disease-free survival ${ }^{[2,3]}$. Mechanisms that contribute to MRD include the existence of mutations such as those found in the BCR-ABL protein kinase domain, variations in stem cell expression, and drug resistance resulting from exposure to chemotherapy treatment ${ }^{[4]}$. Another possible compensatory mechanism recently described implicated mitochondria as an energy source for a survival phenotype of residual malignant cells ${ }^{[5]}$.

High mitochondrial DNA (mtDNA) copy numbers in residual NHL cells were associated with increased citrate synthase and isocitrate dehydrogenase expressions from the same MRD cells. This phenotype was characteristic of unique MRD cells that survived following exposure to chemotherapy ${ }^{[5,6]}$. The findings suggested that alterations in the energy production pathway might have occurred leading to survival of residual malignant cells after exposure to chemotherapy.

We hypothesize that these residual malignant cells are unique and different from primary malignant cells that typically utilize glycolysis as their primary energy source. To address our hypothesis, we exposed residual malignant cells to siRNA targeted against citrate synthase and isocitrate dehydrogenase and measured the effect on mtDNA and lactate dehydrogenase (LDH) as markers for cell integrity.

\section{Methods}

The in-vitro study was designed to emulate what residual tumor cells typically experienced when MRD is present in children and adolescents following chemotherapy for NHL. NHL cells were exposed to chemotherapy followed by isolation of surviving cells for further manipulations. The initial experiments focused on optimizing the siRNA transfection parameters followed by assays for mtDNA and LDH assessments.

\section{Transfection optimization}

Multiple cell lines representing various NHL types were initially tested to identify the feasibility and proof-of-concept for a consistent cell line for transfection experiments. Cell lines were obtained from ATCC (Manassas, VA): ACH-2 (T-cell), U-937 (Monocytic), U-1 (Monocytic), Ramos (B-cell), and H-9 (T-cell) lymphoma cell lines. Cells were processed and maintained in culture as previously noted ${ }^{[5,6]}$. To optimize transfection, the cells were first exposed to positive and negative (non-target) control siRNAs in various concentrations and exposure times. From all of the cell culture experiments, the Ramos cell line provided consistent and reproducible transfection results. Therefore for this initial feasibility study, Ramos cells were identified as the NHL cells for subsequent experiments. Clinical specimens from children with MRD have previously been identified and analyzed for mtDNA copy numbers, which established an in-vivo relationship ${ }^{[5,6]}$. With the positive control silencing expression of GAPDH and the negative control having no known targets in the human genome, an optimal condition in a 24-well plate format, at a concentration of 100,000 cells/well at 48 hours post-transfection resulted in maximum suppression of GADPH relative to GAPDH expression in non-transfected Ramos cells (Table 1). Expression of the housekeeping gene, $\beta$-actin, was used to normalize expression of GADPH.

Table 1A. Reaction quotient values of GAPDH siRNA transfected Ramos cells relative to GAPDH expression in non-transfected Ramos cells (6-well plate format)

\begin{tabular}{llll}
\hline Hrs. post-transfection & Cells/well & & \\
\hline & 200,000 & 400,000 & 600,000 \\
24 & 68 & 72 & 64 \\
48 & 62 & 58 & 59 \\
72 & 64 & 61 & 57 \\
\hline
\end{tabular}


Table 1B. Reaction quotient values of GAPDH siRNA transfected Ramos cells relative to GAPDH expression in non-transfected Ramos cells (12-well plate format)

\begin{tabular}{llll}
\hline Hrs. post-transfection & Cells/well & & \\
\hline & 100,000 & 200,000 & 300,000 \\
24 & 54 & 48 & 56 \\
48 & 42 & 39 & 49 \\
72 & 38 & 41 & 44 \\
\hline
\end{tabular}

Table 1C. Reaction quotient values of GAPDH siRNA transfected Ramos cells relative to GAPDH expression in non-transfected Ramos cells (24-well plate format)

\begin{tabular}{llll}
\hline Hrs. post-transfection & Cells/well & & \\
\hline & 50,000 & 100,000 & 150,000 \\
24 & 32 & 25 & 35 \\
48 & 28 & 16 & 32 \\
72 & 31 & 21 & 41 \\
\hline
\end{tabular}

Note. GADPH expression was normalized in both transfected and non-transfected cells by the housekeeping gene. The Tables demonstrate optimization conditions for the transfection experiments with siRNA.

\section{SiRNA transfection}

Ramos cells were transfected via a standard protocol as per the sureFECT Transfection Reagent Kit (Qiagen, Frederick, MD). One day prior to transfection, 100,000 cells/well were seeded in a 24-well plate with $300 \mu \mathrm{L}$ of RPMI-1640 1X (Mediatech Incorporated; Herndon VA) supplemented with 10\% fetal bovine serum (Sigma-Aldrich, St. Louis, MO), 2\% sodium bicarbonate, $1 \%$ glucose, $1 \%$ sodium pyruvate, and 1\% penicillin/streptomyocin. On the day of transfection, 30 nM siRNA (Sabio Sciences; Frederick, MD) was added to $40 \mu \mathrm{L}$ of Opti-MEM I Reduced Serum Media (Invitrogen; Carlsbad, CA) and mixed gently on the Max Rotator (Barnstead-Lab Line; Melrose Park, IL) at a speed setting of 4 (approximately $500 \mathrm{rpm}$ ) for 5 minutes at room temperature. $40 \mu \mathrm{L}$ of SureFECT Transfection Reagent (Sabio Sciences; Frederick, MD) was then added to the Opti-MEM Medium forming the siRNA-SureFECT mix that was gently agitated on the Max Rotator at a speed setting of 4 for 20 minutes at room temperature. $80 \mu \mathrm{L}$ of the siRNA-SureFECT mix was then added to the cells with $300 \mu \mathrm{L}$ of normal growth media followed by a gentle mix as noted above for 20 minutes at room temperature. Cells were then incubated at $37^{\circ} \mathrm{C}$ in a humidified atmosphere containing $5 \% \mathrm{CO}_{2}$ for 48 hours.

48-hours after initial transfection, the efficiency of knock down in target genes, as well as controls, was assessed via real-time PCR ${ }^{[6]}$. Four different siRNA plasmids, labeled A, B, C and D, were designed to silence expression by identifying a unique sequence in target gene mRNA, as outlined in the previously mentioned SureFECT transfection Kit manual. Fifteen combinations (single, double, triple or all), with a final concentration of $30 \mathrm{nM}$, were tested in order to determine the most effective siRNA cocktail. The cycling parameters utilized were previously noted along with melt curve analysis to confirm specificity ${ }^{[5]}$. All transfections were performed in duplicate with real-time analysis run in triplicate (Figure 1).

\section{Chemotherapy exposure, lactate dehydrogenase and mtDNA assessments}

Ramos cells were exposed to doxorubicin-HCL (500 nM) (Biomol Internationals; Plymouth Meeting, PA) and vincristine sulfate (12nM) (MP Biomedicals; Solon, OH) separately, for one hour as previously described ${ }^{[5]}$. Following washing and 
removal of drug, the surviving Ramos cells were maintained in culture for one week with washing and media replacement every third day. Surviving cells were maintained in culture, and transfected with siRNAs against citrate synthase and isocitrate dehydrogenase expressions as described below, for a total of three weeks. Control Ramos cells were also exposed to chemotherapy agents but the cells were not transfected with siRNA.

Over the three-week post-exposure period, supernatant aliquots were removed on days 9, 13, 17, and 21 to assess lactate dehydrogenase using the Lactate Assay Kit (BioVision; Mountain View, CA) by measuring the production of L(+) -lactate through fluorescence. mtDNA copy numbers were also measured on days 3, 7, 11, 15 and 19, previously described ${ }^{[6]}$. Cell viability was assessed every other day as an indicator of overall cellular functioning via Trypan blue exclusion method as previously described ${ }^{[5]}$.

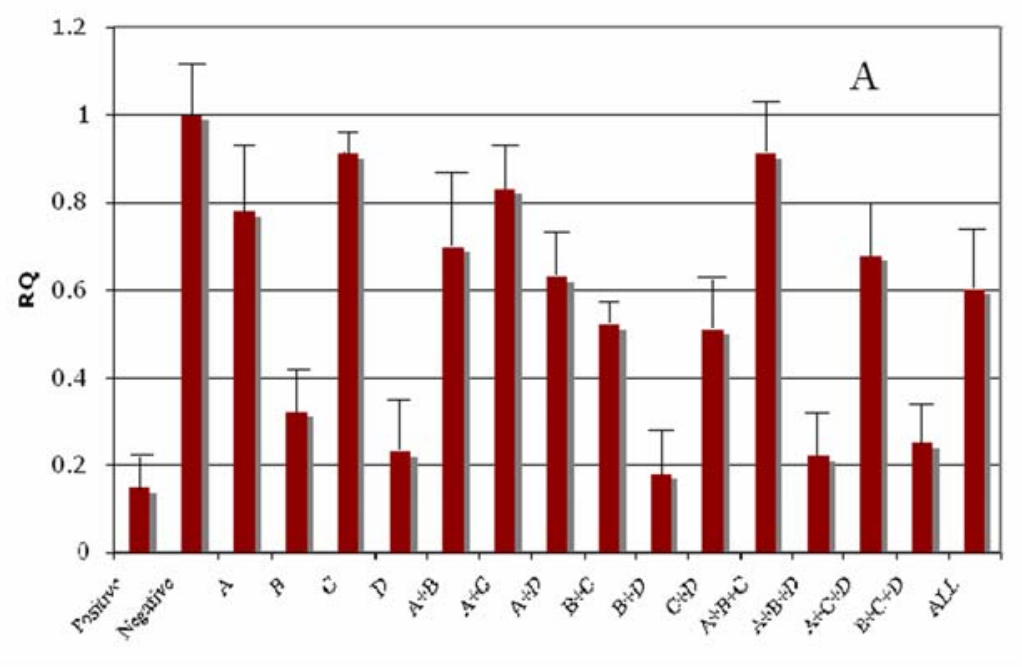

A) Reaction Quotient (RQ) values for siRNA design knocking down expression of citrate synthase. RQ values are calculated as citrate synthase expression levels relative to expression of GAPDH in non-transfected Ramos cells.

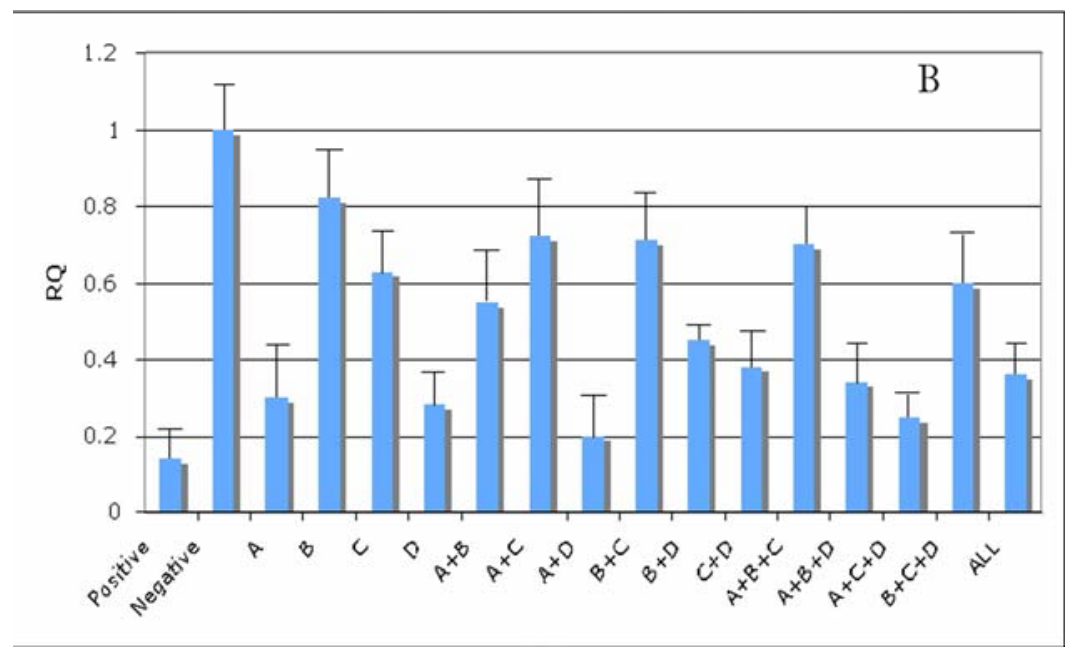

B) RQ values for siRNA design knocking down expression of isocitrate dehydrogenase. RQ values are calculated as isocitrate dehydrogenase levels relative to expression of GAPDH in non-transfected Ramos cells.

Figure 1. Optimization of transfection conditions

Note. Ramos cells were treated with chemotherapy agents as previously outlined prior to transfection in order to be labeled as being residual cells. 


\section{Outcome measures and data analysis}

Expression of the enzyme of interest was assessed relative to GADPH expression in non-transfected Ramos cells and was presented as a Reaction Quotient (RQ) value with 1 being the maximal value observed. The RQ value closest to 0 signified the most effective siRNA cocktail and was later utilized to silence expression of target genes. Student t-tests were run for the LDH and mtDNA analyses with $P$-values less than 0.05 considered significant. The LDH and mtDNA analyses were calculated against the non-transfected cells for comparison after transfection for the genes of interest.

\section{Results}

\section{Lactate levels post-transfection}

Lactate production was assessed following transfection on days 9, 13, 17 and 21, as previously outlined. There was an observed significant decrease in lactate production with increasing days in culture for the cells transfected with citrate synthase siRNA $(P=0.02)$ when compared to lactate production in non-transfected cells. There was also a decrease in the cells transfected with isocitrate dehydrogenase siRNA $(P=0.12)$ and in the cells transfected with the combination siRNA $(P=0.09)$. The decrease in lactate, however, was not as significant as with the citrate synthase siRNA alone. The non-transfected cells exhibited an increase in lactate production over the course of the cell culture period highlighting the differences between the transfected and non-transfected Ramos cells as it pertains to activity in the glycolytic pathway (Figure 2).

Figure 2. Lactate Dehydrogenase Measurements for Treated Ramos cells. Cells were transfected after seven days in culture silencing expression of gene of interest. Note. Labels identify genes that were silenced. Both indicate that both genes of interest were silenced and None indicates no transfection

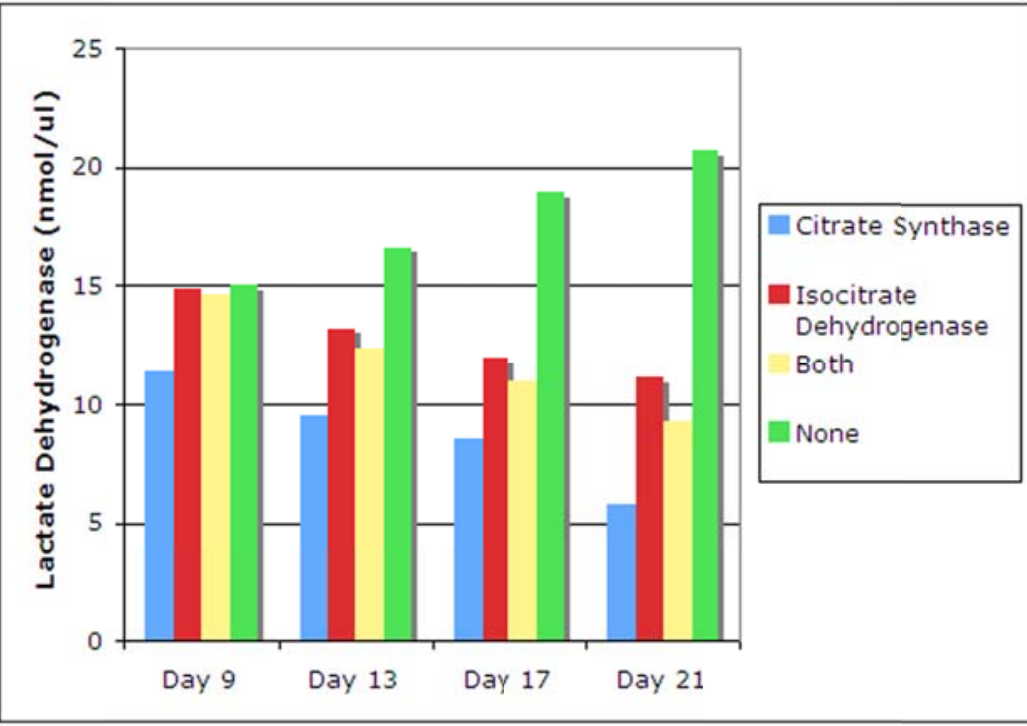

\section{mtDNA copy numbers post- transfection}

There was an increase in mtDNA copy numbers/cell in the non-transfected Ramos cells with increasing days in culture based on previous results representing surviving and recovering malignant cells after chemotherapy exposure ${ }^{[5]}$. The observation reflects the baseline for MRD cells. Ramos cells transfected with citrate synthase siRNA resulted in significant decreased mtDNA copy numbers following transfection when compared to non-transfected cells $(P=0.03)$. The cells transfected with isocitrate dehydrogenase siRNA demonstrated a decrease in mtDNA copy numbers although not as significant $(P=0.22)$. When both citrate synthase- and isocitrate dehydrogenase-siRNA were combined and transfected together, mtDNA copy numbers were decreased more than isocitrate dehydrogenase siRNA alone but not more than citrate synthase siRNA $(P=0.14)$. The control experiments with the non-transfected cells demonstrated that mtDNA copy numbers remained unchanged and stable (Figure 3). 

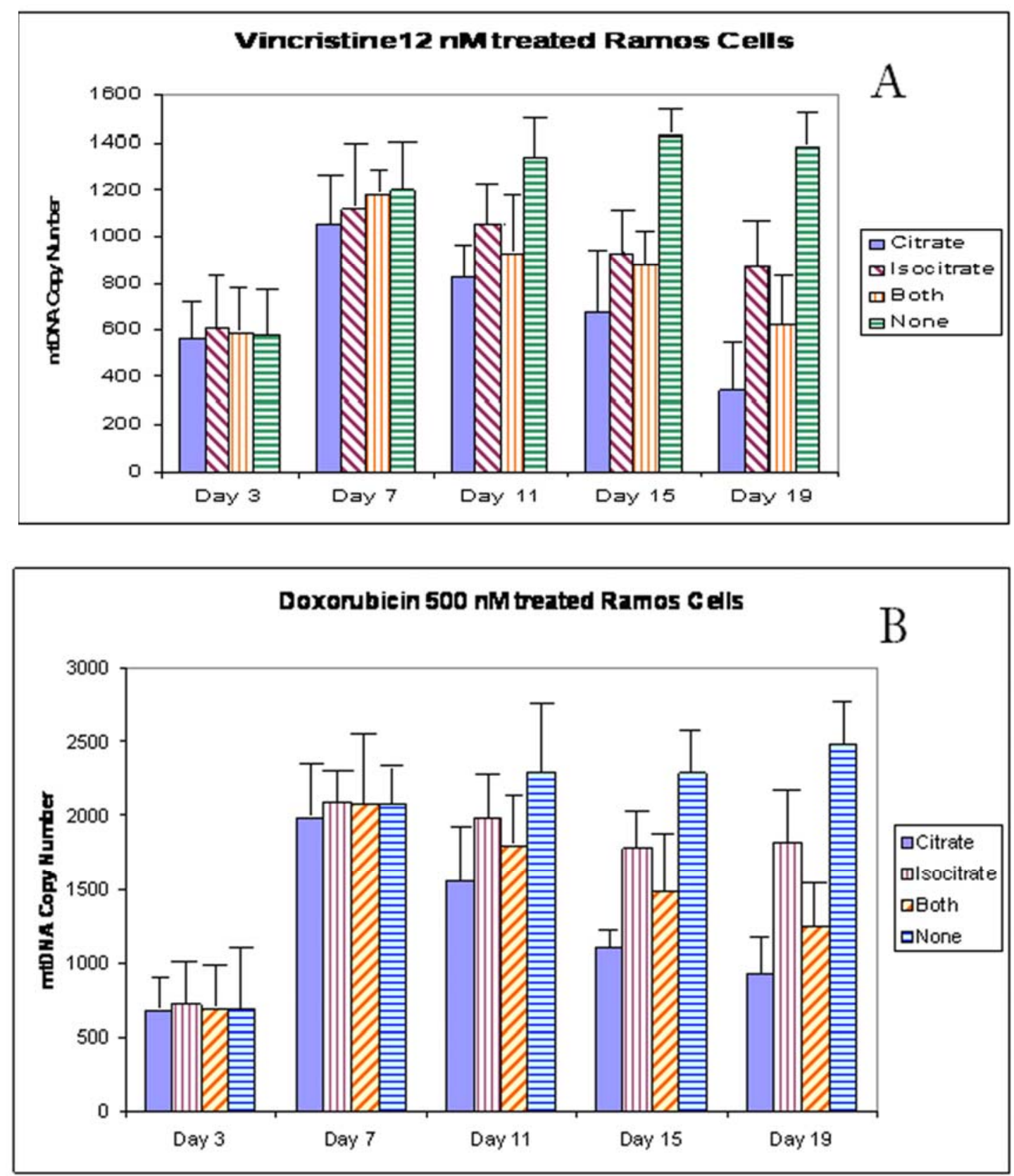

Figure 3. Mitochondria DNA (mtDNA) Copy Numbers of Treated Cells After siRNA Transfection. A) mtDNA Copy number for Vincristine $12 \mathrm{nM}$ treated Ramos cells. Cells were transfected after seven days in culture silencing expression of gene of interest. B) mtDNA copy number for Doxorubicin $500 \mathrm{~nm}$ treated Ramos cells. Cells were transfected after seven days in culture silencing expression of gene of interest.

Note. Labels identify genes that were silenced. Both indicate that both genes of interest were silenced and None indicates no transfection.

\section{Discussion}

While children and adolescents treated for NHL have a relatively good prognosis, those who continue to have MRD detected in blood or bone marrow may continue to be at risk for recurrence of their disease. Recently, blood and/or bone marrow specimens showing MRD also had high mtDNA copy numbers. In-vitro studies further demonstrated that residual NHL cells with high mtDNA copy numbers also had elevated citrate synthase and isocitrate dehydrogenase expressions. 
The current study was undertaken to identify the effects on treatment-exposed NHL cells by inhibiting citrate synthase and isocitrate dehydrogenase expression. The data demonstrated that mtDNA copy numbers decrease following siRNA transfection targeting citrate synthase and isocitrate dehydrogenase. These findings may be an important observation in understanding the survival phenotype of residual lymphoma cells exposed to chemotherapy. The importance of targeting citrate synthase and isocitrate dehydrogenase lies in the energy production shift that typically occurs from oxidative phosphorylation to aerobic glycolysis in malignant cells including the proliferative capacity ${ }^{[7,8]}$. As part of the Krebs cycle, citrate synthase initiates the cycle and serves as a "gate keeper" and thus an important component in the overall production of cellular energy. By silencing citrate synthase expression in the residual malignant cells, mtDNA copy numbers are decreased and revert to a phenotype resembling naive Ramos cells, which might be more easily targeted for treatment. While the observations suggest a mechanism linking citrate synthase and isocitrate dehydrogenase to mtDNA integrity, the results are limited by the in-vitro Ramos cells used which were exposed to chemotherapy. The Ramos cell line was used initially because other cell lines did not consistently demonstrate transfection results. Future assays are planned to determine optimal conditions for the other NHL cells lines. Additional assays will need to be performed in real-time or RNA-preserved cells from MRD-positive blood and/or bone marrow specimens. The decrease in LDH production in the citrate synthase siRNA cells suggested that these cells were possibly shifting away from an energy pathway typically associated with cancer cells. Limitations of these findings might be associated with the siRNA which may have hindered the capacity of the NHL cells to produce sufficient energy via the lactate production pathway. The findings are limited to the effects found on the Ramos cell line however further optimized conditions will need to be completed on other NHL cell lines and immortalized pediatric NHL cells to determine the spectrum of the suggested findings. Finally, the decrease mtDNA copy number associated with a corresponding decrease in LDH was in contrast to our hypothesis. We expected that a decrease in mtDNA copy number would lead to increase in lactate production, as the surviving malignant cell would shift its energy production pathway from the OXPHOS to lactate-based pathway. Our findings support a theory that following the silencing of citrate synthase and isocitrate dehydrogenase, the recovered malignant cells could produce sufficient energy without resorting to the use of lactate. The findings are limited by the assumption that the OXPHOS pathway provides the required energy for cell survival since citrate synthase is a key component in the ATP production pathway and silencing citrate synthase expression would disrupt this production pathway. The fact that cells remain viable following citrate synthase knockdown and the concurrent decrease in lactate production suggested that residual cells may have an energy capacity store or there are internal factors that confer survival in spite of low energy outputs via the OXPHOS or anaerobic pathways.

The study exposed NHL cells to chemotherapy and harvested residual surviving malignant cells to test a siRNA model targeting citrate synthase and isocitrate dehydrogenase. The decrease in mtDNA copy numbers accompanying citrate synthase inhibition supports the theory that alterations in residual cells could be attributed to changes in the production of ATP and the functioning of the OXPHOS pathway ${ }^{[8]}$. Our previous studies identified increased mitochondrial DNA copy numbers associated with positive MRD status suggesting that the mitochondrial, as an energy source, might contribute to the survival of residual cells following chemotherapy exposure ${ }^{[5]}$. Building on these findings, citrate synthase, isocitrate dehydrogenase and succinate dehydrogenase inhibition in residual malignant cells could alter production of ATP and the functioning of the OXPHOS pathway ${ }^{[8]}$. These findings need further validation to test targeted - siRNA against specific enzymes to effect residual malignant cells.

We speculate that mtDNA copy number changes are related to the citrate synthase and isocitrate dehydrogenase pathways, which have not been previously reported based on preliminary observations ${ }^{[6]}$. The data are supported by previous work demonstrating relationships between mtDNA copy number and suppression of other genes in the Krebs cycle ${ }^{[6,9-11]}$. Inhibition by siRNA previously showed that dysregulation of mtDNA copy number and expression may have an adverse effect on mitochondrial function and cell growth ${ }^{[9]}$. Similarly, the relationship between mtDNA copy number and knockdown of succinyl-CoA synthase expression affected mtDNA copy number ${ }^{[10]}$. Finally, succinate dehydrogenase, previously shown to be affect mtDNA copy number and MRD status, inhibition of this enzyme modified histone methylation and mtDNA copy number ${ }^{[5,11]}$. 
In summary, siRNA targeted against citrate synthase and isocitrate dehydrogenase identified unique pathways leading to changes in mtDNA copy numbers in residual chemotherapy-exposed malignant cells. These findings are consistent with the effect on ATP production and potential alterations in the OXPHOS pathway ${ }^{[8,12]}$. Expanding these preliminary findings on the direct on mtDNA copy number on other targeted-siRNA transfected NHL cells in future studies of residual cells could potentially translate into new paradigms for treatment interventions to improve prognosis for children and adolescents impacted by NHL.

\section{Acknowledgements}

The work was support in part by NH grants \#RR026136, \#MD007584, and \#CA121955; and Hawaii Community Foundation Grant \#20061478.

\section{Conflict of interest}

The author declares that there is no conflict of interest statement.

\section{References}

[1] Cairo, M.S., R. Sposto, and S.L. Perkins, Burkitt's and Burkitt-like lymphoma in children and adolescents: a review of the Children's Cancer Group experience. Br J Haematol, 2003. 120: p660-70. PMid: 12588354 http://dx.doi.org/10.1046/j.1365-2141.2003.04134.x

[2] Attarbaschi, A., et al., Minimal residual disease values discriminate between low and high relapse risk in children with B-cell precursor acute lymphoblastic leukemia and an intrachromosomal amplification of chromosome 21: the Austrian and German acute lymphoblastic leukemia Berlin-Frankfurt-Munster (ALL-BFM) trials. J Clin Oncol, 2008.26:p3046-50. PMid: 18565891 http://dx.doi.org/10.1200/JCO.2008.16.1117

[3] Cavé, H., et al., Clinical significance of minimal residual disease in childhood acute lymphoblastic leukemia. European Organization for Research and Treatment of Cancer--Childhood Leukemia Cooperative Group. N Engl J Med, 1998.339:p591-98. PMid: 9718378

[4] Izraeli, S. and D. Waldman, Minimal residual disease in childhood acute lymphoblastic leukemia: current status and challenges. Acta Haematol, 2004.112:p34-39. PMid: 15179003 http://dx.doi.org/10.1159/000077558

[5] Kusao, I., et al., Chemotoxicity recovery of mitochondria in non-Hodgkin lymphoma resulting in minimal residual disease. Pediatr Blood Cancer, 2008.51:p193-97. http://dx.doi.org/10.1002/pbc.21545

[6] Kusao, I., D. Troelstrup, and B. Shiramizu, Possible mitochondria-associated enzymatic role in non-Hodgkin lymphoma residual disease. Cancer Growth Metastasis, 2008:p3-8.

[7] Garber, K., Energy boost: the Warburg effect returns in a new theory of cancer. J Natl Cancer Inst, 2004.96(24):p1805-6. PMid: 15601632 http://dx.doi.org/10.1093/jnci/96.24.1805

[8] Frezza, C. and E. Gottlieb, Mitochondria in cancer: not just innocent bystanders. Semin Cancer Biol, 2009.19:p4-11. PMid:19101633 http://dx.doi.org/10.1016/j.semcancer.2008.11.008

[9] Jeng, J.Y., et al., Maintenance of mitochondrial DNA copy number and expression are essential for preservation of mitochondrial function and cell growth. J Cell Biochem, 2008.103(2):p347-57. PMid:18072287 http://dx.doi.org/10.1002/jcb.21625

[10] Miller, C., et al., The interplay between SUCLA2, SUCLG2, and mitochondrial DNA depletion. Biochim Biophys Acta, 2011. 1812(5):p625-9. PMid: 21295139

[11] Cervera, A.M., et al., Inhibition of succinate dehydrogenase dysregulates histone modification in mammalian cells. Mol Cancer, 2009.8:p89. PMid:19849834 http://dx.doi.org/10.1186/1476-4598-8-89

[12] Semenza, G.L., Oxygen-dependent regulation of mitochondrial respiration by hypoxia-inducible factor 1. Biochem J 2007.405:p1-9. PMid: 17555402 